\title{
Effectiveness of High Energy Advanced Laser Therapy Along With Mobilization Techniques in Treatment of Adhesive Capsulitis of the Shoulder: Randomized Controlled Trial
}

Mohammed Mojahid Hussen ${ }^{1}$, Dr. Pashupati Chaudhary ${ }^{2}$, Chandan Kumar ${ }^{3 *}$

${ }^{1}$ Physiotherapist, Department of Orthopedics, B P Koirala Institute of Health Sciences, Dharan, Nepal

${ }^{2}$ Professor, Department of Orthopedics, B P Koirala Institute of Health Sciences, Dharan, Nepal

${ }^{3}$ Associate Professor- Department of Physiotherapy, School of Allied Health Sciences, Sharda University Noida, India

*Corresponding author: Chandan Kumar

Abstract

Purpose: Shoulder pain is the third most common disorder of musculoskeletal system affecting 15-20\% of the general population. Among these Adhesive capsulitis or Frozen shoulder is the most common. The purpose of the study was to find out the effectiveness of High Energy Advanced Laser Therapy (HEALTH) along with Mobilization techniques on improving pain and functional ability in patients with Adhesive capsulitis of shoulder. Methods: Subjects diagnosed with Adhesive capsulitis \& refer for Physiotherapy treatment were recruited in this study. Subjects willing to be the part of study were divided into two groups. This Interventional study was conducted to assess effectiveness of physiotherapy treatment between two groups. In Group A, subjects were intervened by HEALTH (High Energy Advanced Laser Therapy) and Mobilization techniques along with supervised exercises for 4 weeks. The other Group $\mathrm{B}$ with 15 patients were treated with Mobilization techniques and supervised active exercises only. Beside these both the groups were also doing conventional active exercises for Adhesive capsulitis on regular basis twice a day. All the patients underwent intervention for four weeks and were assessed shoulder ROM, pains using Numerical Pain Rating Scale (NPRS) of total 40 points and Shoulder Pain and Disability Index (SPADI) of total 130 points before and after treatment. The collected data were converted in percentage score and entered at Microsoft Excel 2007 for statistical analysis. Descriptive statistics were calculated along with graphical and tabular presentations. For inferential statistics Student t- test and paired t-test were used to find out the significant difference between inter and intra groups respectively. Results: Out of 30 patients $19(63.33 \%)$ female and $11(36.66 \%)$ male with the mean and standard deviation of age was $51.33 \pm 8.81$ years. The total SPADI score in percentage before and after treatment of both the groups were $70.45 \pm 10.60$ to $41.37 \pm 11.37$ in Group A $(\mathrm{p}<0.001)$ and $69.31 \pm 12.98$ to $53.436 \pm 8.98$ in Group B $(\mathrm{p}<0.006)$ respectively and it was found to be significantly reduced and also statistically significant. Similarly, percentage pain score on NPRS reduced significantly from $76.66 \pm 9.37$ to $38.33 \pm 8.48$ in Group A (p<0.001) and $64.66 \pm 10.08$ to $51.5 \pm 10.64$ in Group B $(\mathrm{p}<0.001)$ respectively. But it was also found that Group A treatment had more reduced in percentages of pain than in post treatment measure by Group B, and it was found to be statistically significant. Conclusion: It was concluded that HEALTH (High Energy Advanced Laser Therapy) and Mobilization techniques in combination is more effective than Mobilization techniques and supervised active exercises only, in reducing pain and improving shoulder functional ability in subjects with Adhesive capabilities of shoulder.

Keywords: Shoulder pain, musculoskeletal system, Physiotherapy.

Copyright @ 2020: This is an open-access article distributed under the terms of the Creative Commons Attribution license which permits unrestricted use, distribution, and reproduction in any medium for non-commercial use (NonCommercial, or CC-BY-NC) provided the original author and source are credited.

\section{INTRODUCTION}

Adhesive capsulitis also known as "Frozen shoulder" is a benign, self-limiting condition of unknown etiology characterized by painful and limited active and passive glenohumeral ROM, in at least two directions, most notably shoulder abduction and external rotation. The frozen shoulder was first described as "Periarthritis" involving the periarticular soft tissues of shoulder by Duplay in 1872. Codman coined the term Frozen shoulder in 1934. J S Navieser coined the term "Adhesive capsulitis" in 1945. In clinical practice it can be hard to differentiate Adhesive capsulitis from other shoulder pathologies. The Adhesive capsulitis is a combination of synovitis and capsular fibrosis, which results in thickening and contracture of the inferior capsule. Frozen shoulder is considered primary if the onset is idiopathic while secondary results from known cause or surgical event. It 
is more prevalent in woman, individuals 35-65 yrs old and in the diabetic population. Sometimes bilateral involvement has been also found. Associated risk factors include: trauma, prolonged immobilization, thyroid disease, stroke, myocardial infarcts and presence of autoimmune disease.

Pain, movement, age and sex component of the disease are as follows:

Pain

- Strong component of night pain

- Pain with rapid or unguarded movement

- Discomfort lying on the affected shoulder

- Pain easily aggravated by movement

Movement

- Global loss of active and passive ROM

- Pain at end range in all directions

Onset

- $\quad>35$ years of age

Sex

- More prevalent in woman

Adhesive capsulitis progresses through three overlapping clinical phases:

- ACUTE/FREEZING/PAINFUL

PHASE:

Gradual onset of shoulder pain at rest with sharp pain at extreme ROM, and throbbing pain at night with sleep disturbances. This may last anywhere from 3-9 months.

- FROZEN/ADHESIVE/STIFFENING PHASE: Pain starts to subside, progressive loss of glenohumeral motion in capsular pattern. Pain is apparently only at extreme of ROM. This phase may start at around four months and last for a year.

- THAWING/RESOLUTION PHASE: Spontaneous progressive improvement in shoulder function and ROM. This may last anywhere from 1-3 years.

Clinical symptoms include pain, limited ROM and altered scapulohumeral rhythm and muscle weakness due to disuse and movements taking in scaption. The incidence of Adhesive capsulitis of shoulder is $2-5 \%$ in general population and $10-20 \%$ in diabetics' patient. The females are more affected than males and are usually seen in age group of 40-60 years. Among the effected individuals some $10 \%$ develop the condition bilaterally.

Even though adhesive capsulitis is believed to be a self-limiting process, it can be severely disabling for months to years. It requires aggressive treatment once the diagnosis is confirmed. Various methods of treatment are available for adhesive capsulitis which helps in maintaining and improving strength of shoulder girdle muscles and functions. It includes thermotherapy, LASER, Ultrasonic therapy, TENS, Isoplanar IFT,
Extracorporeal Shockwave therapy, mobilization, stretching exercises, pendular exercises etc.

A variety of interventions are used by physiotherapists to reduce pain and disability which include exercises, electrotherapy and mobilization techniques. Now a days, High energy advanced laser therapy along with mobilization technique has also became an integral part of treating Adhesive capsulitis.

Joint mobilization techniques are assumed to induce various beneficial effects including neurophysicial and biomechanical effects. Pathological changes cause increase in intraarticular pressure and increases joint restriction. The main objective of mobilization manual therapy is to rescue these changes and improve ROM. In the treatment of Adhesive capsulitis, Maitland mobilization aims in improving range by breaking down adhesions and whereas Mulligan mobilization corrects the malalignment and reduces pain. The approach of Maitland method is based on a graded assessment and treatment system through passive oscillatory movements, rhythmic graded in five levels that vary with the amplitude of the accessory movements normally present in the joints. Various grades of mobilization such as mid range and end range mobilizations are suggested by Maitland and Klatenborn to improve shoulder joint mobility and reduce pain. To regain the normal extensibility of the shoulder capsule and tight soft tissues, mobilization techniques has been advocated more than angular stretching. Mulligan proposes that the Movement with mobilization (MWM) technique has its effect by correcting the positional faults in the joints. Klatenborn [1] mentioned that for any normal motion at the joint to occur, proper joint kinetics is necessary. Adequate capsule extensibility is vital to allow roll sliding to occur between the body surfaces within the joint. Any restriction of the joint capsule or faulty relationship to the joint surface will interface with normal motion. Normal mobility can be stored by either general or specific (Maitland and Mulligan) mobilization technique. The mechanical effect may include the breaking up of adhesions, realigning collagen or increasing fiber glides along with joint arthrokinematics. For the sake of convenience both the technique was used for mobilization as per need of the patient, preferably progressing from Maitland to Mulligan mobilization technique.

\section{Maitland Mobilization}

Physiotherapist applied oscillatory end-range Maitland mobilization grade III or grade IV. The physiotherapist applied oscillatory caudal glides to increase shoulder abduction, posterior glide to increase shoulder flexion \& internal rotation and anterior glide to increase external rotation. 
Mojahid Hussen et al., Sch J App Med Sci, June, 2020; 8(6): 1559-1565

\section{Mulligan Mobilization}

This mobilization technique was described by Mulligan [2], in which the physiotherapist applied passive accessory glide as the patient moved the arm actively in the desired direction within a pain free range. The mobilization was done in 3 sets of 10 repititions in each direction.

\section{High Energy Advanced Laser Therapy}

HEALTH (High Energy Advanced Laser Therapy) is the new concept for assuring health and treating pain and inflammation over several pathologies into the fields of Physiotherapy, Orthopedics, Sports, Dermatology and Beauty Medicine. The equipment used by us was Fiber 5 with $808 \mathrm{~nm}$ wavelength, visible beam $650 \mathrm{~nm} / 5 \mathrm{Mw}$, frequency $1-250 \mathrm{~Hz}$, pulse width $100 \mathrm{~ms}$ and maximum power $5 \mathrm{~W}$. Continuous, pulsed and super pulsed emission modes were available. LASER beam was generated by using optic fiber. 808 $\mathrm{nm}$ is the optimal wavelength for getting in touch with the deepest tissues (Vascular, muscular and tendons). The LASER unit is supposed for a therapeutic use and for a direct irradiation of parts of the human body with infrared laser radiation. Fiber device is suitable for manual application by using $6 \mathrm{~cm}$ applicator. The applicator is made with reference to divergence property of laser source and it is properly calculated with reference to the dimension of the laser beam at a certain distance. High power lasers are able to treat all the same conditions that respond well to cold laser but in less time and more efficacies with more deeply penetrations into the body tissues. The higher the power, the deeper the penetration, allowing for treatment of tissue damage deep within the body. More power leads to shorter treatment times. The greater the

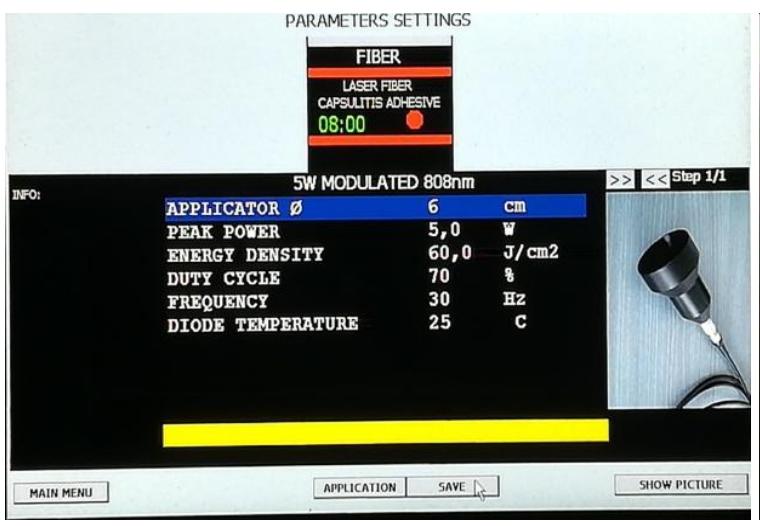

All patients in both the group were given the same instructions and encouragement to practice pendulum/shoulder ROM exercises at home and to participate in daily activities within pain limits.

In patient with adhesive capsulitis the pain intensity was calculated by using Shoulder Pain and Disability Index (SPADI). It was developed to measure current shoulder pain and disability. The latest version of SPADI has items scored on Numeric rating scale which makes it easier to administer and score but the power the more effective the laser is in treating more severe and painful conditions. Energy density is measured in units of joules per square $\mathrm{cm}$.

Adhesive capsulitis is a condition in which shoulder capsule of the Glenohumeral joint becomes inflamed and stiff along with adhesion formation. Mobilization techniques and LASER administration are important interventions. It is known fact that by the time patients come for Physiotherapy treatment, they already have ROM restrictions. This research is done to study the effectiveness High Energy Advanced Laser Therapy along with joint mobilization techniques to restore Glenohumeral joint ROM and pain control.

\section{Benefits/Physiological Effects of Laser Therapy to Users}

- Relieves acute and chronic pain (Analgesic effect)

- Increases the speed, quality and tensile strength of tissue repair

- Increases blood supply (Angiogenesis)

- Stimulates the immune system

- Stimulates nerve function

- Develops collagen and muscle tissue

- Helps generate new and healthy cells and tissue

- Promotes faster wound healing (Activation \& proliferation of fibroblasts)

- Reduced inflammation

- Biostimulation including improved metabolism and increase of cell metabolism

- Anti-edematous effects

- Alteration of action potential

- Activation of phagocytes and macrophages

- Altered endogenous opoid production.

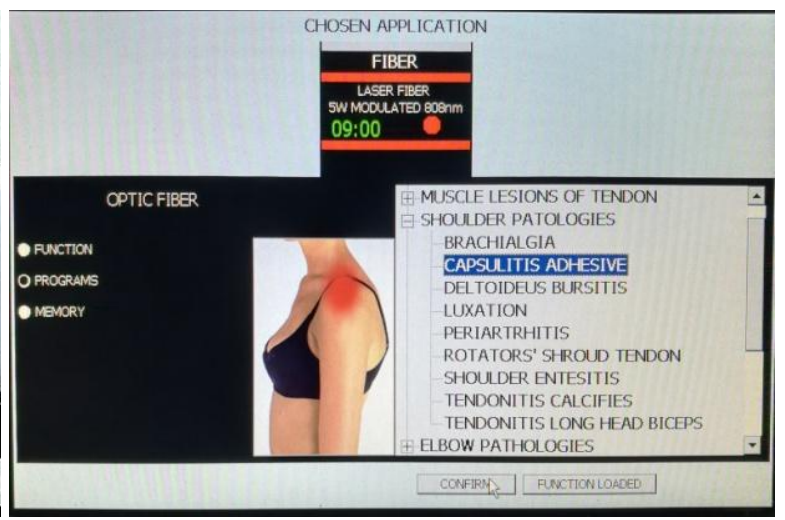

original version of SPADI has each item scored on a Visual Analogue Scale (VAS). Numerical Pain Rating Scale (NPRS) was also used to determine the pain intensity scale.

\section{Materials And Methods}

Patients diagnosed with Adhesive capsulitis who came to Physiotherapy unit, Orthopedics department at BPKIHS for Physiotherapy treatment was recruited in this study. A prospective intervention study 
was carried out with randomized control trial manner for this study. Total 30 patients were enrolled in this study into two groups. The patients came regularly at Physiotherapy unit, Rehabilitation Center, BPKIHS for the physiotherapy treatment. This Interventional study was conducted to assess effectiveness of physiotherapy treatment between two groups. The one group was patient treated with High Energy Advanced Laser Therapy with Mobilization techniques and the other group was treated with mobilization techniques \& supervised active exercises only. In group A with 15 patients, who were intervened by HEALTH (High Energy Advanced Laser Therapy) and Mobilization techniques along with supervised exercises for 4 weeks. The other Group B with 15 patients were treated with Mobilization techniques \& active exercises only. Beside these both the groups were doing conventional active exercises on regular basis twice a day. All the patients underwent intervention for four weeks and were assessed ROM, pains using Numerical Pain Rating Scale (NPRS) of total 40 points and Shoulder Pain and Disability Index (SPADI) of total 130 points before and after treatment. The collected data were converted in percentage score and entered at Microsoft Excel 2007 for statistical analysis. Descriptive statistics were calculated along with graphical and tabular presentations were made. For inferential statistics Student t- test and paired t-test were used to find out the significant difference between inter and intra groups respectively.

\section{Inclusion and Exclusion criteria}

- Age between 35 to 65 years
- $\quad$ Both Gender (both male \& female)

- Having global restriction of shoulder ROM since 2-3 months and.

The inclusion criteria were confirmed medical diagnosis of Adhesive capsulitis of shoulder.

\section{Exclusion Criteria}

- Presence of cognitive or neurological disorders,

- Presence of other injuries and orthopedic disorders associated with shoulder.

\section{RESULTS}

The study was done in Physiotherapy unit, Department of Orthopedics, B P Koirala Institute of Health Sciences, Dharan. The patients were selected according to inclusion and exclusion criteria. The purpose of study was explained to the patient who participated in the study.

Total 30 patients with confirmed diagnosis Adhesive capsulitis were recruited randomly using simple random sampling technique for the research. The participated patients were made clear that the information gathered will be confidential and will be used only for research purpose and consent will be taken. These 30 patients were divided into 2 groups. The 15 patients in Group A were intervened with High Energy Advanced Laser Therapy and Mobilization Techniques and 15 patients in Group B were intervened with mobilization techniques \& active exercises only. The findings were shown in below tables.

Table-1: Comparison between pre and post measurement by groups

\begin{tabular}{|l|l|l|l|l|l|l|}
\hline \multirow{2}{*}{ Measurements score } & \multicolumn{6}{l}{ Mean and standard deviation of scores } \\
\cline { 2 - 7 } & Group A & Group B & \multicolumn{4}{l|}{} \\
\cline { 2 - 7 } & Pre & Post & palue* & Pre & Post & p value \\
\hline Pain SPADI & $76.13 \pm 9.24$ & $45.33 \pm 14.25$ & $<0.001$ & $75.07 \pm 9.56$ & $57.93 \pm 9.40$ & 0.003 \\
\hline Disability SPADI & $66.25 \pm 12.87$ & $38.33 \pm 10.29$ & $<0.001$ & $67.58 \pm 14.93$ & $50.5 \pm 10.94$ & $<0.001$ \\
\hline Total SPADI & $70.45 \pm 10.60$ & $41.37 \pm 11.37$ & $<0.001$ & $69.31 \pm 12.98$ & $53.43 \pm 8.98$ & 0.006 \\
\hline NPRS & $76.66 \pm 9.37$ & $38.33 \pm 8.48$ & $<0.001$ & $64.66 \pm 10.08$ & $51.5 \pm 10.64$ & $<0.001$ \\
\hline
\end{tabular}

* Paired t-test

Table-2: Comparison between post measurements by groups

\begin{tabular}{|l|l|l|l|}
\hline \multicolumn{3}{|c|}{ Mean and standard deviation of post measurement scores } \\
\hline Measurements score & Group A & Group B & p value* \\
\hline Pain SPADI & $45.33 \pm 14.25$ & $57.93 \pm 9.40$ & 0.008 \\
\hline Disability SPADI & $38.33 \pm 10.29$ & $50.5 \pm 10.94$ & 0.004 \\
\hline Total SPADI & $41.37 \pm 11.37$ & $53.43 \pm 8.98$ & 0.003 \\
\hline NPRS & $38.33 \pm 8.48$ & $51.5 \pm 10.64$ & $<0.001$ \\
\hline
\end{tabular}

*Two samples t-test 


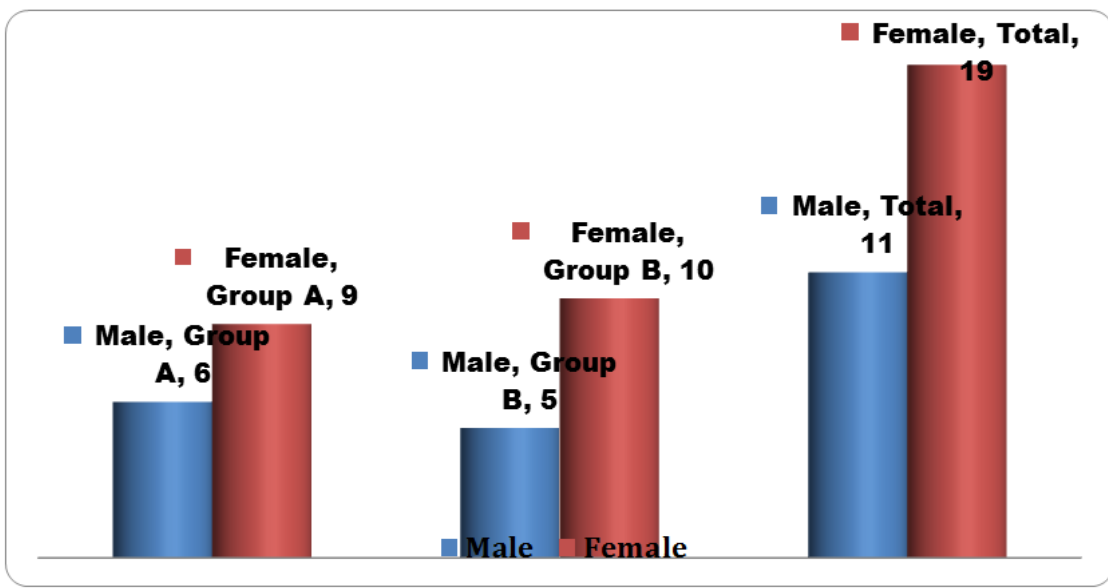

Fig-1: Distribution of gender by groups

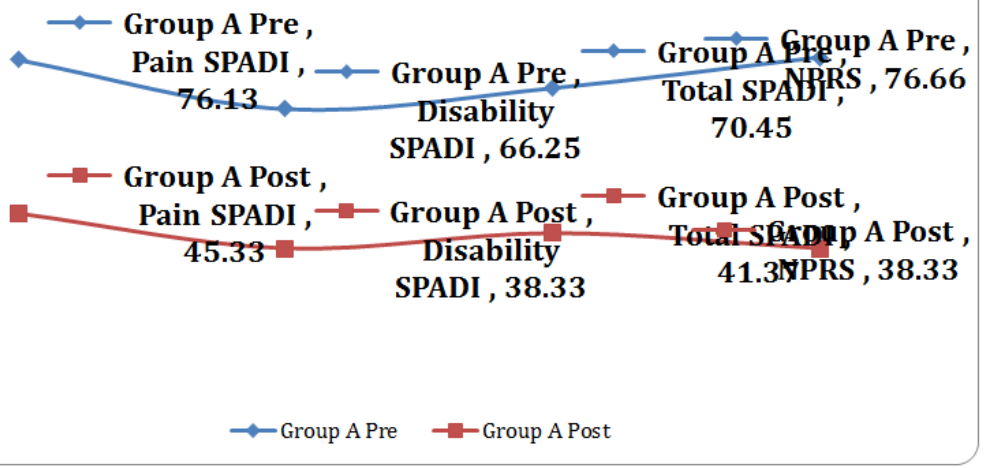

Fig-2: Mean Score of pre and post treatment result of group A

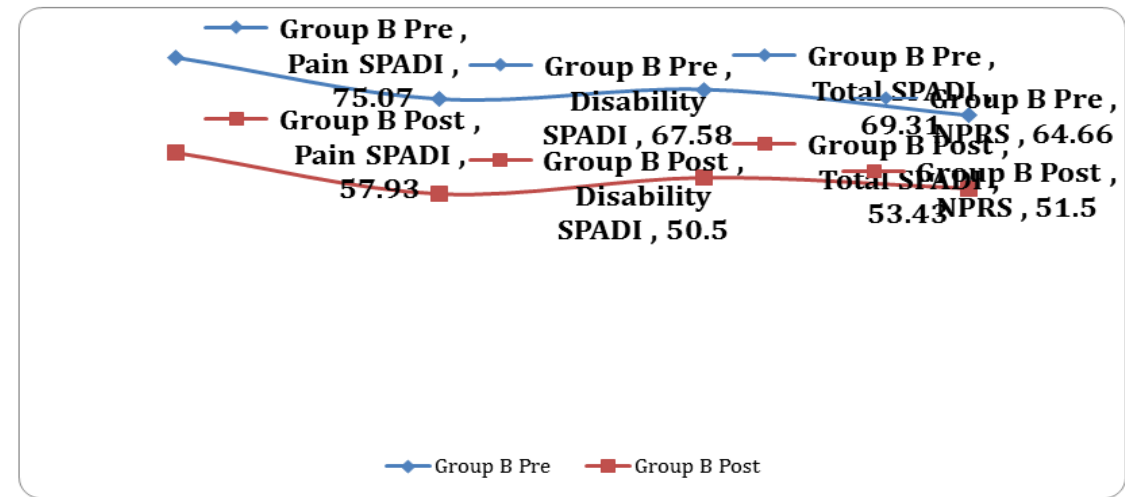

Fig-3: Mean Score of pre and post treatment result of group B

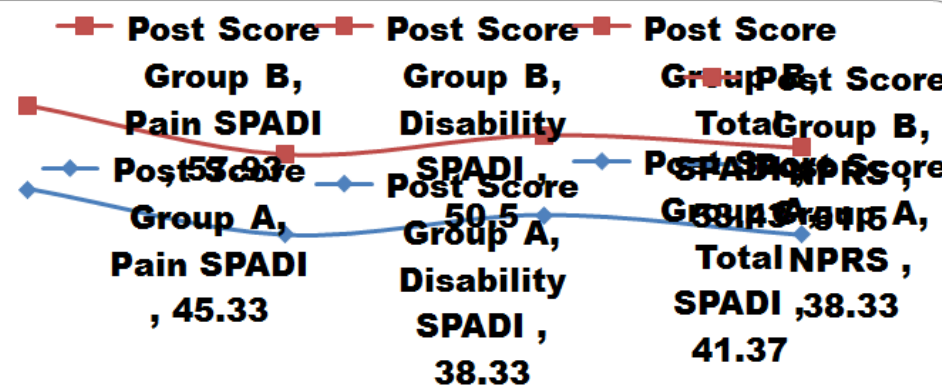

$\neg$ Post Score Group A $\quad-$-Post Score Group B

Fig-4: Mean percentage score of post treatment of both groups 
Out of 30 patients $19(63.33 \%)$ female and 11 $(36.66 \%)$ male with the mean and standard deviation of age was $51.33 \pm 8.81$ years. The total SPADI score in percentage before and after treatment of both the groups were $70.45 \pm 10.60$ to $41.37 \pm 11.37$ in Group A $(\mathrm{p}<0.001)$ and $69.31 \pm 12.98$ to $53.436 \pm 8.98$ in Group B $(\mathrm{p}<0.006)$ respectively and it was found to be significantly reduced and also statistical significant. Similarly percentage pain score on NPRS reduced significantly from $76.66 \pm 9.37$ to $38.33 \pm 8.48$ in Group A $(\mathrm{p}<0.001)$ and $64.66 \pm 10.08$ to $51.5 \pm 10.64$ in Group B $(p<0.001)$ respectively. But it was also found that Group A treatment had more reduced in percentages of pain than in post treatment measure by Group B, and it was found to be statistically significant.

\section{DisCUSSION}

The present study was undertaken to evaluate the efficacy of High Energy Advanced Laser Therapy (HEALTH) and Mobilization techniques in treatment of Adhesive capsulitis of shoulder joint in Group A patients and efficacy of Mobilization techniques \& active shoulder exercises by the patients in Group B. In this study patients were assessed for Shoulder Pain and Functional Disability using SPADI scale (Total 130 points) and NPRS scale (Total 40 points). The therapeutic intervention was planned for 4 weeks for both the groups.

While analyzing the outcome measures of this study, it was observed that Group A showed significant improvement than Group B in both pain and disability parameters. The total SPADI \% score in Group A patients, dropped from $70.05 \pm 10.98$ to $41.33 \pm 11.36$ and NPRS \% score from $72.66 \pm 9.37$ to $38.33 \pm 8.48$, whereas in Group B patients the total SPADI \% score dropped less from $69.31 \pm 12.84$ to $53.436 \pm 8.98$ and NPRS \% score from $64.66 \pm 10.08$ to $51.5 \pm 10.64$. The Disability SPADI score in both the groups dropped more than Pain SPADI score.

The physiological effects of High Energy Advanced Laser Therapy like analgesic effect, angiogenesis, reduced inflammation, improved cell metabolism, activation \& proliferation of fibroblasts, increased tensile strength of tissue repair etc helped the patients in Group A to recover well from pain and disability, compared to Group B patients who were treated by Mobilization techniques \& active exercises only. The findings will shed more light on the benefit of High Energy Advanced Laser Therapy as an adjunct treatment to exercise in the management of shoulder disorder.

Nicholson compared pain and ROM of shoulder joint in two groups who received Mobilization along with exercises and the other group treated with active exercises only, and found that Mobilization group had lesser pain and joint stiffness than control group.
Kalternborn [3] mentions that for any normal motion at the joint to occur, proper joint kinetics is necessary. Adequate capsule extensibility is necessary to allow roll \& sliding to occur between the articular surfaces within the joint. Any restriction of the joint capsule or faulty relationship to the joint surface will interfere with normal motion. The mechanical effect of Mobilization technique may help in breaking of adhesions, realigning collagen and increasing fiber glide. Joint mobilization techniques are assumed to induce various beneficial effects. The neurophysiologic effect is based on the stimulation of peripheral mechanoceptors and the inhibition of nociceptors. Furthermore, mobilization techniques are supposed to increase or maintain joint mobility by inducing changes in synovial fluid, enchanced exchange between synovial fluid and cartilage matrix, and increased synovial fluid turnover.

In a routine physiotherapy clinic, management of various musculoskeletal shoulder disorders is multifaceted and treatment includes a broad spectrum of physiotherapeutic options such as progressive strengthening exercises, strapping, electrotherapy, lowgrade joint manipulation/mobilization therapy, acupuncture, advice and education. However, compared to other treatment options, exercise therapy which includes joint and soft-tissue mobilization, stretching/flexibility, range of motion and gradual strengthening exercises is the mainstay of rehabilitation protocol for Adhesive capsulitis. There are wide variety of intervening technique used in physiotherapy for the treatment of Adhesive capsulitis, one better than another. Among those we found in our study that Group A patients who were treated by High Energy Advanced Laser Therapy and Mobilization techniques responded well than the Group A patients who were treated by Mobilization techniques \& active exercises only.

The limitation of this study includes small sample size, shorter intervention period (as compared to the longer recovery period in patients with Adhesive capsulitis), muscle strength, shoulder ROM and scapulohumeral rhythm were not considered in outcome. As there are lot of factors responsible for recovery in patients with Adhesive capsulitis, studies of larger sample size, with longer intervention period and follow up study are needed to confirm our findings. Subgroup analysis is not included in this study to investigate whether HEALTH (High Energy Advanced Laser Therapy) works differently in different age groups.

\section{Conclusion}

The result of this study can be concluded that HEALTH (High Energy Advanced Laser Therapy) and Mobilization techniques in combination is more effective than conventional Mobilization techniques \& active exercises only, in reducing pain and improving 
shoulder functional ability in patients diagnosed as Adhesive capsulitis of shoulder.

\section{REFERENCES}

1. Klatenborn FM. Manual therapy for the extremity joints. Oslo. Norway: Olaf Norlis Bokhandel: 1976.
2. Mulligan BR. Mobilization with movement. J Manual Manipulative Ther.1993:1:154-156.

3. Kaltenborn FM, Evjenth O, Morgan D. Manual mobilization of the extremity joints: basic examination and treatment techniques. Olaf Norlis; 1989. 